\title{
C. CAnUto
}

\section{Eigenvalue approximations by mixed methods}

RAIRO - Analyse numérique, tome 12, no 1 (1978), p. 27-50.

<http://www.numdam.org/item?id=M2AN_1978_12_1_27_0>

(C) AFCET, 1978, tous droits réservés.

L'accès aux archives de la revue «RAIRO - Analyse numérique » implique l'accord avec les conditions générales d'utilisation (http://www.numdam.org/ legal.php). Toute utilisation commerciale ou impression systématique est constitutive d'une infraction pénale. Toute copie ou impression de ce fichier doit contenir la présente mention de copyright.

\section{Numdam}

Article numérisé dans le cadre du programme

Numérisation de documents anciens mathématiques

http://www.numdam.org/ 


\title{
EIGENVALUE APPROXIMATIONS BY MIXED METHODS (*) (i)
}

\author{
by C. Canuto (2)
}

Communiqué par P.-A. Raviart

\begin{abstract}
We study an abstract eigenvalue problem related to a saddle-point formulation, and then we discretize it, obtaining error bounds for eigenvalues and eigenfunctions. We apply our results to the solution of fourth order problems by mixed finite element methods.
\end{abstract}

\section{INTRODUCTION}

We are interested in the following eigenvalue problem:

Find $\lambda \in \mathbf{R}$ and $(u, \psi) \in V \times W$ such that

$$
\left.\begin{array}{ll}
a(u, v)+b(v, \psi)=0, & \forall v \in V, \\
-b(u, \varphi)=\lambda(\psi, \varphi)_{H}, & \forall \varphi \in W
\end{array}\right\}
$$

(where $V, W, H$ are real Hilbert spaces with $W \subseteq H, a(u, v)$ and $b(v, \varphi)$ are continuous bilinear forms on $V \times V$ and $V \times W$ respectively). Such a problem suggests an abstract scheme for numerical approximation of spectral boundary-value problems for elliptic operators, by means of finite elements of mixed type.

We recall that in the last years the use of mixed methods for solving steadystate problems has been studied by a large number of authors: here, we only mention the abstract works by Babuška [1] and Brezzi [7], the papers by Babuška-Oden-Lee [3], Raviart-Thomas [17], Thomas [18] for Second Order Problems, and by Brezzi-Raviart [8], Ciariet-Raviart [9], Glowinski [11] for Fourth Order Problems; further references can be found in these papers. For the finite element approximation of eigenvalue problems in the compact selfadjoint case, the main references are the paper by Birkhoff-de BoorSwartz-Wendroff [5] and the book by Stang-Fix [19], whose ideas have largely inspired our work; for the nonselfadjoint case we mention the works by Babuška-Aziz [2], Bramble-Osborn [6], Fix [10] and Osborn [16].

(*) Reçu le 5 août 1977.

(') This work was partially supported by the C.N.R.-G.N.A.F.A., and suggested by Prof. F. Brezzi during a series of seminars held at the Istituto Matematico del Politecnico, Turin, in December 1976.

${ }^{(2)}$ Istituto Matematico del Politecnico di Torino. 
Recently Babuška-Osborn [4] have studied a mixed finite element scheme to approximate the eigenvalues of a Second Order operator with discontinuous coefficients.

In this paper we consider a finite dimensional approximation of Problem (1), deriving general error estimates for eigenvalues and eigenfunctions. The abstract functional framework we present here (we refer to Brezzi-Raviart [8] both for notations and for results) is particularly well-suited for the numerical treatment of Fourth Order Problems. Applications to Second Order Problems require a slightly modified version of our theory, which will be presented in a forthcoming note; a third paper, due to P. G. Gilardi, will concern computational aspects of our problem, and numerical tests performed at the "Centro di Calcolo del Politecnico" of Turin. Finally we observe that a finite element approximation of spectral boundary-value problems can be carried out using hybrid methods instead of mixed ones; convergence results and error estimates will appear in a further paper.

An outline of this paper is as follows. In paragraph 1 we state the abstract problem and derive some significant properties for eigenvalues and eigenfunctions, while paragraph 2 contains the corresponding finite dimensional problem. In paragraph 3 we estimate the approximation error for eigenvalues by means of a min-max and a max-min principle; paragraph 4 is devoted to the error bounds for eigenfunctions, in the different norms occurring in our formulation. In the last section we apply our results to the numerical solution of the spectral problem for a clamped plate, using Miyoshi's and Hermann-Johnson's mixed schemes.

\section{STATEMENT OF THE PROBLEM AND ABSTRACT RESULTS}

Let $V$ and $W$ be two infinite dimensional Hilbert spaces, with norms $|\cdot|_{V}$ and $\|.\|_{W}$. If $V^{\prime}$ and $W^{\prime}$ are their respective dual spaces, we denote by $\langle., .\rangle_{V}$ and $\langle\langle., .\rangle\rangle_{W}$ the corresponding duality forms.

Let $a(.,$.$) and b(.,$.$) be two bilinear continuous forms on V \times V$ and $V \times W$ respectively. We set

$$
|a|=\sup _{u, v \in V} \frac{a(u, v)}{|u|_{V}|v|_{V}}
$$

and we assume that the form $a(.,$.$) is symmetric and V$-coercive, i. e. there exists a constant $\alpha>0$ such that

$$
a(v, v) \geqq \alpha|v|_{V}^{2}, \quad \forall v \in V .
$$

We also assume that there exists a constant $\beta>0$ such that

$$
\sup _{v \in V} \frac{b(v, \varphi)}{|v|_{V}} \geqq \beta\|\varphi\|_{W}, \quad \forall \varphi \in W
$$


and we set

$$
\|b\|=\sup _{v \in V, \varphi \in W} \frac{b(v, \varphi)}{|v|_{V}\|\varphi\|_{W}} .
$$

Moreover, let $H$ be a real Hilbert space, with inner product $(., .)_{H}$ and norm $|\cdot|_{H}$ such that

$$
W \subseteq H \text { with compact imbedding and dense immage. }
$$

By identifying the dual space $H^{\prime}$ with $H$, we have $H \subseteq W^{\prime}$ with continuous imbedding, and $\langle\langle\varphi, \eta\rangle\rangle_{W}=(\varphi, \eta)_{H}, \forall \varphi \in H, \forall \eta \in W$.

We consider the following eigenvalue problem:

(EP) Find the eigenvalues $\lambda \in \mathbf{R}$ and the eigenfunctions $(u, \psi) \in V \times W$ such that

$$
\begin{array}{ll}
a(u, v)+b(v, \psi)=0, & \forall v \in V, \\
-b(u, \varphi)=\lambda(\psi, \varphi)_{H}, & \forall \varphi \in W .
\end{array}
$$

Problem (EP) now stated can be reduced to an ordinary eigenvalue problem for a compact, symmetric, positive operator on $H$. The simplest way to do this is the following.

For every $g \in H$, the problem:

Find $(u, \psi) \in V \times W$ such that

$$
\left.\begin{array}{ll}
a(u, v)+b(v, \psi)=0, & \forall v \in V \\
-b(u, \varphi)=(g, \varphi)_{H}, & \forall \varphi \in W
\end{array}\right\}
$$

has, in hypotheses (1.1)-(1.4), a unique solution such that

$$
|u|_{V}+\|\psi\|_{W} \leqq \mathrm{M}|g|_{H}
$$

with $M=M(\alpha,|a|, \beta)$ (see Brezzi [7], Th. 1.1 and Prop. 1.1). As a consequence, the mapping

$$
g \in H \mapsto(u, \underset{v}{\Downarrow}) \in V \times W \mapsto \underset{\gamma}{\|} \in W
$$

is continuous, and since the imbedding $W \subseteq H$ is compact, the operator

$$
\left.\begin{array}{c}
T: H \rightarrow H \\
T g=\psi
\end{array}\right\}
$$

is compact. Moreover, we easily check that $T$ is symmetric, positive and injective. Since problem (EP) does not admit the null eigenvalue, it is clear that $\mu$ is an eigenvalue of $T$ if and only if $\lambda=1 / \mu$ is an eigenvalue of (EP), and $\psi \in W$ is an eigenvector of $T$ if and only if there exists $u \in V$ (necessarily unique) sucht that $(u, \psi) \in V \times W$ is an eigenvector of (EP).

From the spectral theory in Hilbert spaces, we have 
THEOREM 1: Problem (EP) admits an infinite and divergent sequence of real strictly positive eigenvalues, each of finite multiplicity

$$
\left\{\lambda_{l}\right\}_{l=1}^{\infty}
$$

(we agree that eigenvalues are ordered in increasing way and repeated according to their multiplicity), and a corresponding sequence of eigenfunctions

$$
\left\{\left(u_{l}, \psi_{l}\right)\right\}_{l=1}^{\infty}
$$

such that the system $\left\{\psi_{l}\right\}_{l=1}^{\infty}$ is orthonormal complete with respect to the inner product in $H$.

Property 1.1: With respect to the inner product in $V$

$$
(u, v)_{a}=a(u, v), \quad \forall u, v \in V
$$

(equivalent to the inner product associated to the norm $|v|_{V}$ by our hypotheses), the system

$$
\left\{\frac{u_{l}}{\sqrt{\lambda_{l}}}\right\}_{l=1}^{\infty}
$$

is orthonormal, not necessarily complete, in $V$.

Proof: In fact, we have by (1.5)-(1.6)

$$
a\left(u_{l}, u_{m}\right)=-b\left(u_{m}, \psi_{l}\right)=\lambda_{m}\left(\psi_{m}, \psi_{l}\right)_{H} .
$$

In the following, we shall call $U$ the closed submanifold of $V$ spanned by the system (1.12).

REMARK 1.1: Results just stated may be confirmed and improved, by recognizing in problem (EP) an eigenvalue problem for a bilinear coercive form on $W$. To this purpose, for every $\varphi \in W$, we call $u_{\varphi} \in V$ the unique solution of the problem

$$
a\left(u_{\varphi}, v\right)+b(v, \varphi)=0, \quad \forall v \in V
$$

which exists by the coerciveness of the form $a$ on $V$. By (1.2) we have

and by (1.3):

$$
\alpha\left|u_{\varphi}\right|_{V}^{2} \leqq a\left(u_{\varphi}, u_{\varphi}\right) \leqq\|b\|_{i}\left|u_{\varphi}\right|_{V}\|\varphi\|_{W}
$$

that is

$$
\beta\|\varphi\|_{W} \leqq \sup _{v \in V} \frac{b(v, \varphi)}{|v|_{V}} \leqq|a|\left|u_{\varphi}\right|_{V}
$$

$$
\frac{\beta}{|a|}\|\varphi\|_{W} \leqq\left|u_{\varphi}\right|_{V} \leqq \frac{\|b\|}{\alpha}\|\varphi\|_{W}, \quad \forall \varphi \in W .
$$


We can define the following bilinear symmetric form on $W \times W$ :

$$
\mathscr{B}(\varphi, \eta)=-b\left(u_{\varphi}, \eta\right)=a\left(u_{\varphi}, u_{\eta}\right)
$$

By our assumptions and by (1.15), $\mathscr{B}$ is continuous on $W \times W$ and coercive on $W$ :

$$
\mathscr{B}(\varphi, \varphi) \geqq \alpha \frac{\beta^{2}}{|a|^{2}}\|\varphi\|_{W}^{2}, \quad \forall \varphi \in W .
$$

Then problem (EP) is equivalent to the following:

(EP') Find $\lambda \in \mathbf{R}$ and $\psi \in W$ such that

$$
\mathscr{B}(\psi, \varphi)=\lambda(\psi, \varphi)_{H}, \quad \forall \varphi \in W,
$$

while the operator $T$ is characterized by the formula

$$
\mathscr{B}(T g, \varphi)=(g, \varphi)_{H}, \quad \forall g \in H, \quad \forall \varphi \in W .
$$

Hence, from the spectral theory for bilinear coercive forms in Hilbert spaces, we obtain that:

(i) $\operatorname{Im} T$ is dense in $W$;

(ii) the system $\left\{\psi_{l} / \sqrt{\lambda_{l}}\right\}_{l=1}^{\infty}$ is orthonormal complete in $W$ with respect to the inner product induced by $\mathscr{B}(.,$.$) ;$

(iii) the following characterization holds

$$
\varphi \in W \Leftrightarrow \sum_{l=1}^{\infty} \lambda_{l}\left(\varphi, \psi_{l}\right)_{H}^{2}<+\infty
$$

COROLlary 1.1: The subspace $U$ coincides with the space of all the solutions $u \in V$ of $(1.14)$ when $\bar{\varphi}$ runs through $W$, and it is topologically isomorphic to $W$

Proof: It follows immediately from (1.15) and (iii).

Since problem (EP) is equivalent to an eigenvalue problem for a compact symmetric operator, its eigenvalues and eigenfunctions can be characterized by a min-max principle. To this purpose, set

$$
E=\{(u, \psi) \in V \times W \mid a(u, v)+b(v, \psi)=0, \forall v \in V\}
$$

and note that by (ii) and corollary 1.1, we have

$(u, \psi) \in E \Leftrightarrow\left(u=\sum_{i=1}^{\infty} \alpha_{i} u_{i}\right.$ and $\psi=\sum_{i=1}^{\infty} \alpha_{i} \psi_{i} \quad$ with $\left.\sum_{i=1}^{\infty} \lambda_{i} \alpha_{i}^{2}<+\infty\right) ;$ 
now define on $E$ the Rayleigh quotient

$$
R(u, \psi)=\frac{a(u, u)}{(\psi, \psi)_{H}}
$$

Remark 1.2: For every $(u, \psi) \in E$, we have

$$
R(u, \psi)=R_{\mathscr{B}}(\psi)
$$

where $R_{\mathscr{B}}(\psi)=\mathscr{B}(\psi, \psi) /(\psi, \psi)_{H}$ is the Rayleigh quotient on $W$ of the bilinear form (1.16).

As a consequence, the following proposition holds:

Proposition 1.1 (Min-Max Principle): The stationary points of $R$ on $E$ are precisely the eigenfunctions of problem (EP) and the values of $R$ at such points are the corresponding eigenvalues. Moreover, for every $l \in N_{*}$ :

$$
\lambda_{I}=\min _{\substack{S_{l} \subseteq E \\ \operatorname{dim} S_{l}=l}} \max _{(u, \psi) \in S_{l}} R(u, \psi)
$$

the minimum being taken over all l-dimensional subspaces of $E$, and being achieved on the subspace $E_{l}$ spanned by the first l eigenvectors $\left\{\left(u_{1}, \psi_{1}\right), \ldots,\left(u_{l}, \psi_{l}\right)\right\}$.

Proof: The proposition translates in terms of the space $V \times W$ and the quotient $R(u, \psi)$ the well known min-max principle for the quotient $\boldsymbol{R}_{\mathscr{B}}(\psi)$ (see for a proof Strang-Fix [19], Sec. 6.1).

Conversely, a max-min principle holds for inverse eigenvalues $\mu_{l}=1 / \lambda_{l}$.

Proposition 1.2: Defining on $H$ the quotient

$$
Q(\varphi)=\frac{(T \varphi, \varphi)_{H}}{(\varphi, \varphi)_{H}}
$$

we have for every $l \in N_{*}$ :

$$
\mu_{l}=\max _{\substack{\bar{s}_{l} \subseteq H \\ \operatorname{dim} \bar{S}_{l}=l}} \min _{\varphi \in \bar{S}_{l}} Q(\varphi)
$$

the maximum being taken over all l-dimensional subspaces $\bar{S}_{l} \subseteq H$ and being achieved on the subspace $\bar{E}_{l}$ spanned by the first l eigenfunctions $\left\{\psi_{1}, \ldots, \psi_{l}\right\}$.

Proof: It is the same as that of the min-max principle.

Finally, we restate problem (EP) in a different functional framework, in view of its numerical approximation. To this end, we consider two real Hilbert spaces $\tilde{V}$ and $\tilde{W}$, with norms $\|\cdot\|_{\tilde{V}}$ and $\|\cdot\|_{\tilde{W}}$, such that

$$
\begin{aligned}
& \tilde{V} \subseteq V \text { and } W \subseteq \tilde{W} \subseteq H \quad \text { with continuous imbeddings, } \\
& W \text { is dense in } \tilde{W} \text {. }
\end{aligned}
$$

R. A. I. R. O. Analyse numérique/Numerical Analysis 
We assume that there exist a continuous bilinear form $\tilde{b}(.,$.$) on \tilde{V} \times \tilde{W}$ and a constant $\tilde{\beta}>0$ such that

$$
\begin{gathered}
\tilde{b}(v, \varphi)=b(v, \varphi), \quad \forall v \in \tilde{V}, \quad \forall \varphi \in W, \\
\sup _{v \in \tilde{V}} \frac{\tilde{b}(v, \varphi)}{\|v\|_{\tilde{V}}} \geqq \tilde{\beta}\|\varphi\|_{\tilde{W}}, \quad \forall \varphi \in \tilde{W} .
\end{gathered}
$$

We set

$$
\|\tilde{b}\|=\sup _{v \in \tilde{V}, \varphi \in \tilde{W}} \frac{\tilde{b}(v, \varphi)}{\|v\| \tilde{v}\|\varphi\|_{\tilde{W}}}
$$

and consider the following eigenvalue problem:

$(\widetilde{\mathrm{EP}})$ Find the eigenvalues $\lambda \in \mathbf{R}$ and the eigenfunctions $(u, \psi) \in \tilde{V} \times \tilde{W}$ such that

$$
\begin{array}{ll}
a(u, v)+\tilde{b}(v, \psi)=0, & \forall v \in \tilde{V}, \\
-\tilde{b}(u, \varphi)=\lambda(\psi, \varphi)_{H}, & \forall \varphi \in \tilde{W} .
\end{array}
$$

The following proposition links together problems $(\mathrm{EP})$ and $(\widetilde{\mathrm{EP}})$.

Proposition 1.3: Assume that problem (1.7) is regular with respect to $H$, in the sense that for every $g \in H$ the solution $(u, \psi) \in V \times W$ is such that $u \in \tilde{V}$. Then every eigensolution $\left(\lambda_{l} ; u_{l}, \psi_{l}\right)$ of $(\mathrm{EP})$ is an eigensolution of $(\widetilde{\mathrm{EP}})$.

Proof: It follows from Theorem 2 of Brezzi-Raviart [8].

\section{APPROXIMATION OF PROBLEM (EP)}

Given two finite dimensional spaces $V_{h}$ and $W_{h}$ such that

$$
V_{h} \subseteq \tilde{V}, \quad W_{h} \subseteq \tilde{W}
$$

we assume that there exists a constant $\tilde{\gamma}>0$ such that

$$
\sup _{v_{h} \in V_{h}} \frac{\tilde{b}\left(v_{h}, \varphi_{h}\right)}{\left\|v_{h}\right\|_{\tilde{V}}} \geqq \tilde{\gamma}\left\|\varphi_{h}\right\| \tilde{w}, \quad \forall \varphi_{h} \in W_{h}
$$

and consider the following approximation of problem ( $\widetilde{\mathrm{EP}})$ :

$(\mathrm{EP})_{h}$ Find the eigenvalues $\lambda^{h} \in \mathbf{R}$ and the eigenfunctions $\left(u^{h}, \psi^{h}\right) \in V_{h} \times W_{h}$ such that

$$
\left.\begin{array}{cc}
a\left(u^{h}, v_{h}\right)+\tilde{b}\left(v_{h}, \psi^{h}\right)=0, & \forall v_{h} \in V_{h} \\
-\tilde{b}\left(u^{h}, \varphi_{h}\right)=\lambda^{h}\left(\psi^{h}, \varphi_{h}\right)_{H}, & \forall \varphi_{h} \in W_{h}
\end{array}\right\}
$$


Recall that in the hypotheses (1.2) and (2.2), for every $g \in H$ the problem: Find $\left(u_{h}, \psi_{h}\right) \in V_{h} \times W_{h}$ such that

$$
\left.\begin{array}{cc}
a\left(u_{h}, v_{h}\right)+\tilde{b}\left(v_{h}, \psi_{h}\right)=0, & \forall v_{h} \in V_{h} \\
-\tilde{b}\left(u_{h}, \varphi_{h}\right)=\left(g, \varphi_{h}\right)_{H}, & \forall \varphi_{h} \in W_{h}
\end{array}\right\}
$$

has a unique solution (see Brezzi [7], Prop. 2.1) such that

$$
\left|u_{h}\right|_{V}+\left\|\psi_{h}\right\| \tilde{W} \leqq \tilde{M}|g|_{H}
$$

with $\tilde{M}=\tilde{M}(\alpha,|a|, \tilde{\gamma})$, hence independent of $h$. So, by restricting ourselves to the subspace $W_{h} \subseteq H$, we can define the bounded one-to-one operator

$$
\begin{gathered}
T_{h}: \quad W_{h} \rightarrow W_{h}, \\
T_{h} g_{h}=\psi_{h} .
\end{gathered}
$$

Setting $N_{h}=\operatorname{dim} W_{h}$, from the spectral theory in finite dimensional spaces, we obtain the following:

THEOREM 2.1: Problem (EP) $)_{h}$ admits exactly $N_{h}$ real strictly positive possibly repeated eigenvalues

$$
\left\{\lambda_{l}^{h}\right\}_{l=1}^{N_{h}}
$$

(arranged in increasing order), and $N_{h}$ corresponding eigenfunctions

$$
\left\{\left(u_{l}^{h}, \psi_{l}^{h}\right)\right\}_{l=1}^{N_{h}}
$$

such that the system $\left\{\psi_{l}^{h}\right\}_{l=1}^{N h}$ is an orthonormal basis of $W_{h}$ with respect to the inner product of $\mathrm{H}$.

PROPERTY 2.1: The system

$$
\left\{\frac{u_{l}^{h}}{\sqrt{\lambda_{l}^{h}}}\right\}_{l=1}^{N_{h}}
$$

is orthonormal with respect to the inner product $(., .)_{a}$ induced by $V$ on $V_{h}$.

PROPERTY 2.2: The subspace $U_{h} \subseteq V_{h}$ spanned by system (2.8) coincides with the space of solutions $u_{h} \in V_{h}$ of the problem

$$
a\left(u_{h}, v_{h}\right)+\tilde{b}\left(v_{h}, \varphi_{h}\right)=0, \quad \forall v_{h} \in V_{h}
$$

when $\varphi_{h}$ runs through $W_{h}$.

Now we characterize the approximate eigenvalues. Set

$$
E^{h}=\left\{\left(u_{h}, \psi_{h}\right) \in V_{h} \times W_{h} \mid a\left(u_{h}, v_{h}\right)+\tilde{b}\left(v_{h}, \psi_{h}\right)=0, \forall v_{h} \in V_{h}\right\}
$$


and define on $E^{h}$ the Rayleigh quotient

$$
R_{h}\left(u_{h}, \dot{\psi}_{h}\right)=\frac{a\left(u_{h}, u_{h}\right)}{\left(\psi_{h}, \psi_{h}\right)_{H}}
$$

REMARK 2.1: Though the two quotients $R$ and $R_{h}$ relative to the continuous and approximate problem have the same expression, they must be considered as "different", because they are defined on different spaces $E$ and $E^{h}$, which have no reciprocal inclusion relations.

In analogy with the continuous problem, we have:

Proposition 2.1 (Min-Max Principle): For every $1 \leqq l \leqq N_{h}$ :

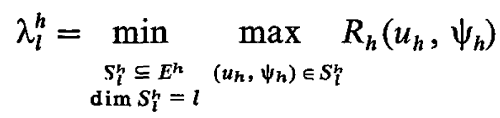

the minimum being achieved on the subspace $E_{l}^{h}$ spanned by the first l eigenvectors $\left\{\left(u_{1}^{h}, \psi_{1}^{h}\right), \ldots,\left(u_{l}^{h}, \psi_{l}^{h}\right)\right\}$.

Proof: Similar to that of proposition 1.1 .

Proposition 2.2 (Max-Min Principle): Defining on $W_{h}$ the quotient

$$
Q_{h}\left(\varphi_{h}\right)=\frac{\left(T_{h} \varphi_{h}, \varphi_{h}\right)_{H}}{\left(\varphi_{h}, \varphi_{h}\right)_{H}}
$$

for every $1 \leqq l \leqq N_{h}$ we have (with $\mu_{l}^{h}=1 / \lambda_{l}^{h}$ ):

$$
\mu_{l}^{h}=\max _{\substack{\overline{S_{i}^{h}} \subseteq W_{h} \\ \operatorname{dim} \overline{S_{l}^{h}}=l}} \min _{\varphi_{h} \in S_{l}^{\tilde{S}}} Q_{h}\left(\varphi_{h}\right)
$$

the maximum being achieved on the-subspace- $\bar{E}_{l}^{h}$ spanned by the-first 1 -igenfunctions $\left\{\psi_{1}^{h}, \ldots, \psi_{l}^{h}\right\}$.

In the following we shall assume that there exist two "interpolation" operators

$$
\pi_{h}: \quad \tilde{V} \rightarrow V_{h} \quad \text { and } \quad r_{h}: \quad \tilde{W} \rightarrow W_{h}
$$

and, for every $v \in \tilde{V}$ and $\varphi \in \tilde{W}$, we denote the corresponding interpolation errors by

$$
\begin{aligned}
& \varepsilon^{h}(v)=\varepsilon^{h}(v ; \quad V)=\left|v-\pi_{h} v\right|_{V}, \\
& \tilde{\varepsilon}^{h}(v)=\tilde{\varepsilon}^{h}(v ; \tilde{V})=\left\|v-\pi_{h} v\right\|_{\tilde{V}}, \\
& \varepsilon^{h}(\varphi)=\varepsilon^{h}(\varphi ; H)=\left|\varphi-r_{h} \varphi\right|_{H}, \\
& \tilde{\varepsilon}^{h}(\varphi)=\tilde{\varepsilon}^{h}(\varphi ; \tilde{W})=\left\|\varphi-r_{h} \varphi\right\|_{\tilde{W}} .
\end{aligned}
$$

vol. $12, \mathrm{n}^{\circ} 1,1978$ 
Moreover, we recall that since $V_{h}$ is finite dimensional, there exists a constant $S(h)>0$ depending on $V_{h}$ such that

$$
\left\|v_{h}\right\|_{\tilde{v}} \leqq S(h) \cdot\left|v_{h}\right|_{V}, \quad \forall v_{h} \in V_{h} .
$$

\section{EIGENVALUES ERROR ESTIMATES}

In this section we shall derive abstract bounds for the error $\left|\lambda_{l}-\lambda_{l}^{h}\right|$.

The structure of Problem $(\widetilde{\mathrm{EP}})$ and of its approximation $(\mathrm{EP})_{h}$ does not generally imply that true eigenvalues are approximated from above, as for standard elliptic problems (see also Remark 2.1). Consequently we must find bounds for both $\lambda_{l}^{h}-\lambda_{l}$ and $\lambda_{l}-\lambda_{l}^{h}$. We proceed in two steps: first, we get an intermediate bound for the former quantity by the min-max principle, and for the latter by the max-min principle. The estimate for $\lambda_{l}-\lambda_{l}^{h}$ is quite rough - as it depends on the lack of smoothness of trial functions - nevertheless in practice it guarantees the convergence of approximate eigenvalues to the exact ones. Then, since such a result will be sufficient - in the next sectionto get an "optimal" bound for $\left|\psi_{l}-\psi_{l}^{h}\right|_{H}$, we establish the basic estimate for $\left|\lambda_{l}-\lambda_{l}^{h}\right|$ in terms of this quantity, obtaining an "optimal" result.

We start by estimating the quantity $\lambda_{l}^{h}-\lambda_{l}$. For every

we define the element

$$
(u, \psi)=\sum_{i=1}^{l} c_{i}\left(u_{i}, \psi_{i}\right) \in E_{l},
$$

$$
(\tilde{u}, \tilde{\psi})=\sum_{i=1}^{l} \lambda_{i} c_{i}\left(u_{i}, \psi_{i}\right) \in E_{l}
$$

so that $\psi=T \tilde{\psi}$ or equivalently $(u, \psi)$ is the solution of the problem

$$
\left.\begin{array}{ll}
a(u, v)+\tilde{b}(v, \psi)=0, & \forall v \in \tilde{V} \\
-\tilde{b}(u, \varphi)=(\tilde{\psi}, \varphi)_{H}, & \forall \varphi \in \tilde{W}
\end{array}\right\}
$$

Then we can define an operator

$$
P_{h}: \quad E_{l} \rightarrow E^{h}
$$

by setting $P_{h}(u, \psi)=\left(P_{h} u, P_{h} \psi\right)=$ solution of the problem

$$
\left.\begin{array}{cc}
a\left(P_{h} u, v_{h}\right)+\tilde{b}\left(v_{h}, P_{h} \psi\right)=0, & \forall v_{h} \in V_{h} \\
-\tilde{b}\left(P_{h} u, \varphi_{h}\right)=\left(\tilde{\psi}, \varphi_{h}\right)_{H}, & \forall \varphi_{h} \in W_{h}
\end{array}\right\}
$$

Consider the quantities

$$
A_{l}^{h}=\max _{(u, \psi) \in \Sigma_{l}}\left|a\left(P_{h} u, P_{h} u\right)-a(u, u)\right|
$$

R.A.I.R.O. Analyse numérique/Numerical Analysis 
and

where

$$
\sigma_{l}^{h}=\max _{(u, \psi) \in \Sigma_{l}}\left|2\left(\psi, \psi-P_{h} \psi\right)_{H}-\left(\psi-P_{h} \psi, \psi-P_{h} \psi\right)_{H}\right|
$$

$$
\Sigma_{l}=\left\{(u, \psi) \in E_{l} \mid \psi \in \bar{\Sigma}_{l}\right\} \quad \text { and } \quad \bar{\Sigma}_{l}=\left\{\left.\psi \in \bar{E}_{l}|| \psi\right|_{H}=1\right\} .
$$

From

$$
a\left(P_{h} u, P_{h} u\right)-a(u, u)=a\left(u-P_{h} u, u-P_{h} u\right)-2 a\left(u, u-P_{h} u\right)
$$

it follows that

$$
A_{l}^{h} \leqq c \max _{(u, \psi) \in \Sigma_{l}}\left|u-P_{h} u\right|_{V}\left\{\left|u-P_{h} u\right|_{V}+\sqrt{\lambda_{l}}\right\} .
$$

On the other hand, since

$$
\sigma_{l}^{h} \leqq \max _{(u, \psi) \in \Sigma_{l}}\left|\psi-P_{h} \psi\right|_{H} \cdot\left(2+\left|\psi-P_{h} \psi\right|_{H}\right)
$$

it is reasonable to assume that

$$
\sigma_{l}^{h} \leqq \frac{1}{2} \text { at least for small } h .
$$

This will be always true in applications. Then:

PROPERTy 3.1: The subspace $P_{h} E_{l} \subseteq E^{h}$ is l-dimensional.

Proof: We must only show that, if $P_{h}\left(u^{*}, \psi^{*}\right)=0$, then $\left(u^{*}, \psi^{*}\right)=0$. If $\psi^{*} \neq 0$, we can put $\left|\psi^{*}\right|_{H}=1$, so

$$
\sigma_{l}^{h} \geqq\left|2\left(\psi^{*}, \psi^{*}-P_{h} \psi^{*}\right)_{H}-\left(\psi^{*}-P_{h} \psi^{*}, \psi^{*}-P_{h} \psi^{*}\right)_{H}\right|=\left|\psi^{*}\right|_{H}^{2}=1
$$

which contradicts (3.9); hence $\psi^{*}=0$ and necessarily $u^{*}=0$.

We can now apply the Min-Max Principle (2.9) to the subspace $P_{h} E_{l}$. Note that, if $(u ; \psi) \in \Sigma_{l}$, we have

$$
\left(P_{h} \psi, P_{h} \psi\right)_{H}=(\psi, \psi)_{H}-2\left(\psi, \psi-P_{h} \psi\right)_{H}+\left(\psi-P_{h} \psi, \psi-P_{h} \psi\right)_{H} \geqq 1-\sigma_{l}^{h}
$$

and hence

$$
\lambda_{l}^{h} \leqq \max _{P_{h} E_{l}} R_{h}\left(P_{h} u, P_{h} u\right) \leqq \frac{1}{1-\sigma_{l}^{h}} \cdot\left[\max _{\Sigma_{l}} a(u, u)+A_{l}^{h}\right]
$$

so that, by Proposition 1.1 and (3.9) (which implies $\left(1-\sigma_{l}^{h}\right)^{-1} \leqq 1+2 \sigma_{l}^{h}$ ), we obtain:

Proposition 3.1: The following estimate holds

$$
\lambda_{l}^{h}-\lambda_{l} \leqq A_{l}^{h}+2 \lambda_{l} \sigma_{l}^{h}+2 A_{l}^{h} \sigma_{l}^{h}
$$

with $A_{l}^{h}, \sigma_{l}^{h}$ bounded as in (3.7), (3.8).

vol. $12, \mathrm{n}^{\circ} 1,1978$ 
REMARK 3.1: This is not the best estimate we are able to derive from the min-max principle for $\lambda_{l}^{h}-\lambda_{l}$ : by a rather technical computation we can give "optimal" bounds for $A_{l}^{h}$ and $\sigma_{l}^{h}$ in the sense that they are of the same order as $a\left(u-P_{h} u, u-P_{h} u\right)$ with respect to $h$. However we omit these proofs, since in the following we shall directly obtain the same "optimal" estimate for the quantity $\left|\lambda_{l}^{h}-\lambda_{l}\right|$.

Now we apply the max-min principles (1.22) and (2.11) to the subspace $\bar{E}_{l}^{h} \subseteq H$ in order to estimate $\mu_{l}^{h}-\mu_{l}$. We have

$$
\mu_{l}^{h} \leqq \min _{\bar{E}_{l}^{h}} Q_{h}\left(\varphi_{h}\right) \leqq \min _{\bar{E}_{l}^{h}} \frac{\left(T \varphi_{h}, \varphi_{h}\right)_{H}}{\left|\varphi_{h}\right|_{H}^{2}}+\max _{\bar{E}_{l}^{h}} \frac{\left(T \varphi_{h}-T_{h} \varphi_{h}, \varphi_{h}\right)_{H}}{\left|\varphi_{h}\right|_{H}^{2}}
$$

and therefore:

Proposition 3.2: The following estimate holds

$$
\mu_{l}^{h}-\mu_{l} \leqq \max _{\bar{E}_{l}^{h}} \frac{\left|i T \varphi_{h}-T_{h} \varphi_{h}\right|_{H}}{\left|\varphi_{h}\right|_{H}}
$$

It is clear that (3.11) together with (3.10) gives a bound for $\lambda_{l}-\lambda_{l}^{h}$; we shall see that in the applications these results imply the convergence of each $\lambda_{l}^{h}$ to the corresponding $\lambda_{l}$.

We shall now give another bound for $\left|\lambda_{l}-\lambda_{l}^{h}\right|$, involving the approximation error $\left|\psi_{l}-\psi_{l}^{h}\right|_{H}$. Recall that $\psi_{l}$ and $\psi_{l}^{h}$ are the $l$ th exact and approximate eigenfunctions of unitary norm; next assume that $\left(\psi_{l}, \psi_{l}^{h}\right)_{H} \neq 0$ and $\left(\psi_{l}^{h}, P_{h} \psi_{l}\right)_{H} \neq 0$ at least for small $h$. By (1.28) with $\varphi=\psi_{l}^{h}$, and by (2.3) with $\varphi_{h}=P_{h} \psi_{l}$, we get

$$
\lambda_{l}=\frac{-\tilde{b}\left(u_{l}, \psi_{l}^{h}\right)}{\left(\psi_{l}, \psi_{l}^{h}\right)_{H}}, \quad \lambda_{l}^{h}=\frac{-\tilde{b}\left(u_{l}^{h}, P_{h} \psi_{l}\right)}{\left(\psi_{l}^{h}, P_{h} \psi_{l}\right)_{H}}
$$

But definition of $P_{h}$ leads to

$$
-\tilde{b}\left(u_{l}^{h}, P_{h} \psi_{l}\right)=a\left(P_{h} u_{l}, u_{l}^{h}\right)=-\tilde{b}\left(P_{h} u_{l}, \psi_{l}^{h}\right)=-\tilde{b}\left(u_{l}, \psi_{l}^{h}\right)
$$

so that

$$
\lambda_{l}-\lambda_{l}^{h}=-\frac{\lambda_{l}}{\left(\psi_{l}^{h}, P_{h} \psi_{l}\right)_{H}}\left(\psi_{l}-P_{h} \psi_{l}, \psi_{l}^{h}\right)_{H}
$$

This formula would already give optimal estimates for $\left|u_{l}-u_{l}^{h}\right|_{v}$ and $\left\|\psi_{l}-\psi_{l}^{h}\right\|_{\tilde{w}}$, once we have shown that $\psi_{l}^{h} \rightarrow \psi_{l}$ in $H$ so that the denominator is close to 1 as $h \rightarrow 0$. But we can proceed: we have

$$
-\left(\psi_{l}-P_{h} \psi_{l}, \psi_{l}^{h}\right)_{H}=\left(\psi_{l}-P_{h} \psi_{l}, \psi_{l}-\psi_{l}^{h}\right)_{H}-\left(\psi_{l}-P_{h} \psi_{l}, \psi_{l}\right)_{B}
$$

R.A.I.R.O. Analyse numérique/Numerical Analysis 
where, by definition of $\lambda_{l}$ :

$$
\begin{aligned}
-\left(\psi_{l}-P_{h} \psi_{l}, \psi_{l}\right)_{H} & =\lambda_{l}^{-1} \tilde{b}\left(u_{l}, \psi_{l}-P_{h} \psi_{l}\right) \\
& =\lambda_{l}^{-1}\left[\tilde{b}\left(u_{l}-P_{h} u_{l}, \psi_{l}-P_{h} \psi_{l}\right)+\tilde{b}\left(P_{h} u_{l}, \psi_{l}-P_{h} \psi_{l}\right)\right]
\end{aligned}
$$

Now by (3.4) we have

$$
\tilde{b}\left(u_{l}-P_{h} u_{l}, \psi_{l}-P_{h} \psi_{l}\right)=\tilde{b}\left(u_{l}-P_{h} u_{l}, \psi_{l}-\varphi_{h}\right), \quad \forall \varphi_{h} \in W_{h}
$$

while by (1.27) and (3.4) we get

$$
\begin{aligned}
\tilde{b}\left(P_{h} u_{l}, \psi_{l}-P_{h} \psi_{l}\right) & =-a\left(u_{l}-P_{h} u_{l}, P_{h} u_{l}\right) \\
& =a\left(u_{l}-P_{h} u_{l}, u_{l}-P_{h} u_{l}\right)+\tilde{b}\left(u_{l}-P_{h} u_{l}, \psi_{l}\right) \\
& =a\left(u_{l}-P_{h} u_{l}, u_{l}-P_{h} u_{l}\right)+\tilde{b}\left(u_{l}-P_{h} u_{l}, \psi_{l}-\varphi_{h}\right)
\end{aligned}
$$

for every $\varphi_{h} \in W_{h}$. Hence

$$
\begin{aligned}
-\left(\psi_{l}-P_{h} \psi_{l}, \psi_{l}^{h}\right)_{H}= & \left(\psi_{l}-P_{h} \psi_{l}, \psi_{l}-\psi_{l}^{h}\right)_{H} \\
& +\lambda_{l}^{-1}\left[a\left(u_{l}-P_{h} u_{l}, u_{l}-P_{h} u_{l}\right)+2 b\left(u_{l}-P_{h} u_{l}, \psi_{l}-\varphi_{h}\right)\right]
\end{aligned}
$$

At last, we have

and

$$
\left|a\left(u_{l}-P_{h} u_{l}, u_{l}-P_{h} u_{l}\right)\right| \leqq|a|\left|u_{l}-P_{h} u_{l}\right|_{V}^{2},
$$

$$
\left|\tilde{b}\left(u_{l}-P_{h} u_{l}, \psi_{l}-\varphi_{h}\right)\right| \leqq\|\tilde{b}\|\left\|u_{l}-P_{h} u_{l}\right\| \tilde{v}\left\|\psi_{l}-\varphi_{h}\right\| \tilde{w}
$$

where, by $(2.16)$ we have

$$
\left\|u_{t}-P_{h} u_{t}\right\|_{\tilde{r}} \leqq\left\|u_{l}-v_{h}\right\| \tilde{v} \pm S(h)\left|v_{h}-P_{h} u_{l}\right|_{V 2} \quad \forall v_{h} \in V_{l} .
$$

Therefore we can state the main result of this section

Proposition 3.3: The following estimate holds

$$
\begin{aligned}
\left|\lambda_{l}-\lambda_{l}^{h}\right| \leqq & \frac{1}{\left|\left(\psi_{l}^{h}, P_{h} \psi_{l}\right)_{H}\right|} \\
& \times\left\{\lambda_{l}\left|\psi_{l}-\psi_{l}^{h}\right|_{H}\left|\psi_{l}-P_{h} \psi_{l}\right|_{H}\right. \\
& +|a|\left|u_{l}-P_{h} u_{l}\right|_{V}^{2}+2|| \tilde{b} \| \tilde{\varepsilon}^{h}\left(\psi_{l}\right) \\
& \left.\times\left[\tilde{\varepsilon}^{h}\left(u_{l}\right)+S(h)\left[\left|u_{l}-P_{h} u_{l}\right|_{V}+\varepsilon^{h}\left(u_{l}\right)\right]\right]\right\} .
\end{aligned}
$$

A supplementary hypothesis yields a similar estimate for $\left|\lambda_{l}-\lambda_{l}^{h}\right|$, which does not involve the constant $S(h)$. To this purpose we define as in Brezzi- 
Raviart [8], Th. 4:

$$
\begin{gathered}
Z_{h}(g)=\left\{v_{h} \in V_{h} \mid-\tilde{b}\left(v_{h}, \varphi_{h}\right)=\left(g, \varphi_{h}\right)_{H}, \forall \varphi_{h} \in W_{h}\right\} \quad \text { for } g \in H, \\
Z_{h}=\left\{v_{h} \in V_{h} \mid \tilde{b}\left(v_{h}, \varphi_{h}\right)=0, \forall \varphi_{h} \in W_{h}\right\}=Z_{h}(0), \\
Z=\{v \in \tilde{V} \mid \tilde{b}(v, \varphi)=0, \forall \varphi \in W\} .
\end{gathered}
$$

Proposition 3.3 bis: If we assume

$$
Z_{h} \subseteq Z
$$

then the following estimate holds

$$
\begin{aligned}
\left|\lambda_{l}-\lambda_{l}^{h}\right| \leqq & \frac{1}{\left|\left(\psi_{l}^{h}, P_{h} \psi_{l}\right)_{H}\right|}\left\{\lambda_{l}\left|\psi_{l}-\psi_{l}^{h}\right|_{H}\left|\psi_{l}-P_{h} \psi_{l}\right|_{H}\right. \\
& \left.+|a| \cdot\left|u_{l}-P_{h} u_{l}\right|_{V}^{2}+c\|\tilde{b}\| \tilde{\varepsilon}^{h}\left(u_{l}\right) \tilde{\varepsilon}^{h}\left(\psi_{l}\right)\right\} .
\end{aligned}
$$

with $c>0$ independent of $h$.

Proof: For any $z_{h} \in Z_{h}$ we get by (3.14)

and hence

$$
b\left(z_{h}, \psi_{l}-\varphi_{h}\right)=0
$$

$$
\tilde{b}\left(u_{l}-P_{h} u_{l}, \psi_{l}-\varphi_{h}\right)=\tilde{b}\left(u_{l}-v_{h}, \psi_{l}-\varphi_{h}\right), \quad \forall v_{h} \in Z_{h}\left(\lambda_{l} \psi_{l}\right), \quad \forall \varphi_{h} \in W_{h} .
$$

Since (see Brezzi-Raviart [8], Th. 4):

$$
\inf _{v_{h} \in Z_{h}\left(\lambda_{l} \psi_{l}\right)}\left\|u_{l}-v_{h}\right\| \tilde{r} \leqq c \tilde{\varepsilon}^{h}\left(u_{l}\right)
$$

we obtain the estimate as in the proof of Proposition 3.3.

\section{EIGENFUNCTIONS ERROR ESTIMATES}

In this section, we shall estimate the approximation errors for eigenfunctions in the norms of $H, \tilde{W}$ and $V$.

No accurate estimate of $\left|\lambda_{l}-\lambda_{l}^{h}\right|$ is needed to obtain an optimal bound for $\left|\psi_{l}-\psi_{l}^{h}\right|_{H}$, but only the convergence of each $\lambda_{j}^{h}$ to $\lambda_{j}$ as $h \rightarrow 0$ (roughly speaking, every true eigenvalue must be far enough from approximated eigenvalues of "different index"); as we already said, this fact will be used to get an optimal estimate for $\left|\lambda_{l}-\lambda_{l}^{h}\right|$. On the contrary the estimates for $\left|u_{l}-u_{l}^{h}\right|_{V}$ and $\left\|\psi_{l}-\psi_{l}^{h}\right\|_{\tilde{w}}$ depend explicitly on the quantity $\left|\lambda_{l}-\lambda_{l}^{h}\right|$.

For the sake of semplicity, we shall only deal with the case of simple eigenvalues, briefly sketching the alternative proofs in the case of repeated eigenvalues. 
We assume that, for every $j \in N_{*}$ :

$$
\lim _{h \rightarrow 0} \lambda_{j}^{h}=\lambda_{j},
$$

which is true in applications, thanks to estimates (3.10)-(3.11).

We start with the term $\left|\psi_{l}-\psi_{l}^{h}\right|_{H}$. Since the system $\left\{\psi_{i}^{h}\right\}_{i=1}^{N h}$ is orthonormal and complete in $W_{h}$ (Th. 2), we can write

$$
P_{h} \psi_{l}=\sum_{j=1}^{N_{h}}\left(P_{h} \psi_{l}, \psi_{j}^{h}\right)_{H} \psi_{j}^{h}
$$

Setting $\beta_{l}^{h}=\left(P_{h} \psi_{l}, \psi_{l}^{h}\right)_{H}$, we first estimate the quantity $\left|P_{h} \psi_{l}-\beta_{l}^{h} \psi_{l}^{h}\right|_{H}$, so that we must consider coefficients $\left(P_{h} \psi_{l}, \psi_{j}^{h}\right)_{H}$ with $j \neq l$. Using the definition of exact and approximate eigenfunction (1.27) and (2.3), and of projection $P_{h}$ (3.4), we get

$$
\begin{aligned}
\lambda_{j}^{h}\left(P_{h} \psi_{l}, \psi_{j}^{h}\right)_{H} & =-\tilde{b}\left(u_{j}^{h}, P_{h} \psi_{l}\right)=a\left(P_{h} u_{l}, u_{j}^{h}\right) \\
& =-\tilde{b}\left(P_{h} u_{l}, \psi_{j}^{h}\right)=-\tilde{b}\left(u_{l}, \psi_{j}^{h}\right)=\lambda_{l}\left(\psi_{l}, \psi_{j}^{h}\right)_{H}
\end{aligned}
$$

whence, adding the term $-\lambda_{l}\left(P_{h} \psi_{l}, \psi_{j}^{h}\right)_{H}$ to both sides, we obtain

$$
\left(P_{h} \psi_{l}, \psi_{j}^{h}\right)_{H}=\frac{\lambda_{l}}{\lambda_{j}^{h}-\lambda_{l}}\left(\psi_{l}-P_{h} \psi_{l}, \psi_{j}^{h}\right)_{H}
$$

Setting

$$
2 d_{l}=\min \left\{\lambda_{l}-\lambda_{l-1}, \lambda_{l+1}-\lambda_{l}\right\}
$$

assumption (A) and the monotonicity of eigenvalues yield, for $h$ small enough

$$
\left|\lambda_{l}-\lambda_{j}^{h}\right| \geqq d_{l}, \quad \forall j \neq l
$$

hence, from (4.1)-(4.2)

$$
\begin{aligned}
\left|P_{h} \psi_{l}-\beta_{l}^{h} \psi_{l}^{h}\right|_{H}^{2} & \leqq\left(\frac{\lambda_{l}}{d_{l}}\right)^{2} \sum_{\substack{j=1 \\
j \neq l}}^{N_{h}}\left(\psi_{l}-P_{h} \psi_{l}, \psi_{j}^{h}\right)_{H}^{2} \\
& \leqq\left(\frac{\lambda_{l}}{d_{l}}\right)^{2}\left|\psi_{l}-P_{h} \psi_{l}\right|_{H}^{2}
\end{aligned}
$$

Now we can turn to our approximation error. We have

$$
\left|\psi_{l}-\psi_{l}^{h}\right|_{H} \leqq\left|\psi_{l}-\beta_{l}^{h} \psi_{l}^{h}\right|_{H}+\left|\beta_{l}^{h}-1\right|
$$

and since we can choose the sign of $\psi_{l}$ so that $\beta_{l}^{h}>0$, we get

$$
\left|\beta_{l}^{h}-1\right|=\left.|| \beta_{l}^{h} \psi_{l}^{h}\right|_{H}-\left|\psi_{l}\right|_{H}|\leqq| \beta_{l}^{h} \psi_{l}^{h}-\left.\psi_{l}\right|_{H}
$$

vol. $12, \mathrm{n}^{\circ} 1,1978$ 
Then (4.3), (4.4), (4.5) give us:

Proposition 4.1: The following estimate holds

$$
\left|\psi_{l}-\psi_{l}^{h}\right|_{H} \leqq 2\left(1+\frac{\lambda_{l}}{d_{l}}\right) \cdot\left|\psi_{l}-P_{h} \psi_{l}\right|_{H}
$$

REMARK 4.1: The error $\left|\psi_{l}-\psi_{l}^{h}\right|_{H}$ can be considered optimal, in the sense that it is of the same order of the approximation error in the same norm for the corresponding steady-state problem.

REMARK 4.2: If $\lambda_{l}$ is an eigenvalue of multiplicity $m>1$, (i.e. if $\left.\lambda_{l}=\lambda_{l+1}=\ldots=\lambda_{l+m-1}\right)$ we define $\beta_{i j}^{h}=\left(P_{h} \psi_{l+j}, \psi_{l+i}^{h}\right)_{H}$ and again obtain

$$
\left|P_{h} \psi_{l+i}-\sum_{j=0}^{m-1} \beta_{i j}^{h} \psi_{l+j}^{h}\right|_{H} \leqq \frac{\lambda_{l}}{d_{l}}\left|\psi_{l+i}-P_{h} \psi_{l+i}\right|_{H}
$$

where now $2 d_{l}=\min \left\{\lambda_{l}-\lambda_{l-1}, \lambda_{m}-\lambda_{l}\right\}$, so that for $i=0, \ldots, m-1$

$$
\left|\psi_{l+i}-\sum_{j=0}^{m-1} \beta_{i j}^{h} \psi_{l+j}^{h}\right|_{H} \leqq\left(1+\frac{\lambda_{l}}{d_{l}}\right)\left|\psi_{l+i}-P_{h} \psi_{l+i}\right|_{H}
$$

If the right side is small enough, the system $\left\{\sum_{j=0}^{m-1} \beta_{i j}^{h} \psi_{l+j}^{h}\right\}_{i=0}^{m-1}$ is linearly independent, so by the Gram-Schmidt process we can get an orthonormal system $\left\{\sum_{j=0}^{m-1} \hat{\beta}_{i j}^{h} \psi_{l+j}^{h}\right\}_{j=0}^{m-1}$ such that again $\left|\psi_{l+i}-\sum_{j=0}^{m-1} \hat{\beta}_{i j}^{h} \psi_{l+j}^{h}\right|_{H}$ can be bounded in terms of the errors $\left|\psi_{l+k}-P_{h} \psi_{l+k}\right|_{H}, k=0, \ldots, m-1$. Moreover if we set $\hat{\psi}_{l+i}=\sum_{j=0}^{m-1} \hat{\eta}_{i j}^{h} \psi_{l+j}$ with $\left\{\hat{\eta}_{i j}^{h}\right\}_{0 \leqq i, j \leqq m-1}=\left\{\hat{\beta}_{r s}^{h}\right\}_{0 \leqq}^{-1}, s \leqq m-1$, we obtain a new orthonormal basis of the eigenspace of $\lambda_{l}$, for which the following estimate-extending (4.6)-holds $(i=0, \ldots, m-1)$ :

$$
\left|\hat{\psi}_{l+i}-\psi_{l+i}^{h}\right|_{H} \leqq c\left(1+\frac{\lambda_{l}}{d_{l}}\right)^{m} \sum_{k=0}^{-1}\left|\psi_{l+k}-P_{h} \psi_{l+k}\right|_{H}
$$

Let us now consider the term $\left|u_{l}-u_{l}^{h}\right|_{v}$; we proceed as for the previous estimate, working with the inner product $(., .)_{a}$ and associated norm $|\cdot|_{a}$ (see Property 1.1). Since the system $\left\{\tilde{u}_{j}^{h}\right\}_{j=1}^{N h}$ with $\tilde{u}^{h}=u_{j}^{h} / \sqrt{\lambda_{j}^{h}}$ is an orthonormal basis of $U_{h}$ (Properties 2.1 and 2.2), we can set

$$
P_{h} u_{l}=\sum_{j=1}^{N_{h}}\left(P_{h} u_{l}, \tilde{u}_{j}^{h}\right)_{a} \tilde{u}_{j}^{h}
$$


where, for any $j \neq l$ we have

$$
\begin{aligned}
\left(P_{h} u_{l}, \tilde{u}_{j}^{h}\right)_{a} & =-\tilde{b}\left(P_{h} u_{l}, \tilde{\psi}_{j}^{h}\right)=-\tilde{b}\left(u_{l}, \tilde{\psi}_{j}^{h}\right) \\
& =\frac{\lambda_{l}}{\sqrt{\lambda_{j}^{h}}}\left(\psi_{l}, \psi_{j}^{h}\right)_{H}=\frac{\lambda_{l}}{\sqrt{\lambda_{j}^{h}}}\left(\psi_{l}-\psi_{l}^{h}, \psi_{j}^{h}\right)_{H}
\end{aligned}
$$

because of the orthogonality of the approximate eigenfunctions. Assumption (A) and the monotonicity of eigenvalues yield, if $h$ is small enough,

$$
\lambda_{j}^{h} \geqq \frac{1}{2} \lambda_{1}, \quad 1 \leqq j \leqq N_{h}
$$

thus, setting $\gamma_{l}^{h}=\left(P_{h} u_{l}, \tilde{u}_{l}^{h}\right)_{a}$, we get

$$
\left|P_{h} u_{l}-\gamma_{l}^{h} \tilde{u}_{l}^{h}\right|_{a}^{2} \leqq 2 \frac{\lambda_{l}^{2}}{\lambda_{1}}\left|\psi_{l}-\psi_{l}^{h}\right|_{H}^{2}
$$

On the other hand, the triangle inequality gives

Now

$$
\left|u_{l}-u_{l}^{h}\right|_{a} \leqq\left|u_{l}-\gamma_{l}^{h} \tilde{u}_{l}^{h}\right|_{a}+\left|\gamma_{l}^{h}-\sqrt{\lambda_{l}^{h}}\right|
$$

$$
\gamma_{l}^{h}=-\tilde{b}\left(P_{h} u_{l}, \tilde{\psi}_{l}^{h}\right)=-\tilde{b}\left(u_{l}, \tilde{\psi}_{l}^{h}\right)=\frac{\lambda_{l}}{\sqrt{\lambda_{l}^{h}}}\left(\psi_{l}, \psi_{l}^{h}\right)_{H}
$$

so that

$$
\begin{aligned}
\left|\gamma_{l}^{h}-\sqrt{\lambda_{l}^{h}}\right|= & \left|\frac{\lambda_{l}\left(\psi_{l}, \psi_{l}^{h}\right)_{H}-\lambda_{l}^{h}}{\sqrt{\lambda_{l}^{h}}}\right| \leqq \frac{\lambda_{l}}{\sqrt{\lambda_{l}^{h}}}\left|\left(\psi_{l}-\psi_{l}^{h}, \psi_{l}^{h}\right)_{H}\right| \\
& +\frac{\left|\lambda_{l}-\lambda_{l}^{h}\right|}{\sqrt{\lambda_{l}^{h}}} \leqq \sqrt{\frac{2}{\lambda_{1}}}\left\{\lambda_{l}\left|\psi_{l}-\psi_{l}^{h}\right|_{H}+\left|\lambda_{l}-\lambda_{l}^{h}\right|\right\} .
\end{aligned}
$$

Collecting (4.8), (4.9), (4.7) and recalling the equivalence of the norms $|\cdot|_{a}$ and $|\cdot|_{r}$, we can state

Proposition 4.2: The following estimate holds

$$
\left|u_{l}-u_{l}^{h}\right|_{V} \leqq c\left\{\left|u_{l}-P_{h} u_{l}\right|_{V}+2 \sqrt{\frac{2}{\lambda_{1}}} \lambda_{l}\left|\psi_{l}-\psi_{l}^{h}\right|_{H}+\sqrt{\frac{2}{\lambda_{1}}}\left|\lambda_{l}-\lambda_{l}^{h}\right|\right\}
$$

with $c$ independent of $h$ and $l$.

Remark 4.3: Following the suggestions of Remark 4.2 we can prove a result similar to (4.10) when $\lambda_{l}$ has multiplicity $m>1$.

Finally, by standard techniques, we estimate $\left\|\psi_{l}-\psi_{l}^{h}\right\|_{\tilde{w}}$. We have

$$
\left\|\psi_{l}-\psi_{l}^{h}\right\|_{\tilde{W}} \leqq\left\|\psi_{l}-\varphi_{h}\right\| \tilde{w}_{\tilde{W}}+\left\|\varphi_{h}-\psi_{l}^{h}\right\|_{\tilde{W}}, \quad \forall \varphi_{h} \in W_{h} .
$$


Hypothesis (2.2) gives the existence of $\bar{v}_{h} \in V_{h}$ (depending on $\varphi_{h}$ ) such that

$$
\begin{aligned}
\tilde{\gamma}\left\|\varphi_{h}-\psi_{l}^{h}\right\|_{\tilde{w}} & \leqq \frac{\tilde{b}\left(\bar{v}_{h}, \psi_{l}^{h}-\varphi_{h}\right)}{\left\|\bar{v}_{h}\right\|_{\tilde{v}}} \\
& =\frac{\tilde{b}\left(\bar{v}_{h}, \psi_{l}-\varphi_{h}\right)}{\left\|\bar{v}_{h}\right\|_{\tilde{v}}}+\frac{a\left(u_{l}-u_{l}^{h}, \bar{v}_{h}\right)}{\left\|\bar{v}_{h}\right\|_{\tilde{v}}} .
\end{aligned}
$$

By (1.23) and the continuity of the forms $a$ and $b$, we obtain:

Proposition 4.3: The following estimate holds

$$
\left\|\psi_{l}-\psi_{l}^{h}\right\|_{W} \leqq c\left\{\tilde{\varepsilon}^{h}\left(\psi_{l}\right)+\left|u_{l}-u_{l}^{h}\right|_{V}\right\}
$$

with $c$ independent of $h$ and $l$.

\section{APPLICATIONS}

We apply our abstract results to the numerical approximation of the following eigenvalue problem

$$
\left.\begin{array}{ll}
\Delta^{2} w=\lambda w & \text { in } \Omega, \\
w=\frac{\partial w}{\partial n}=0 & \text { on } \Gamma
\end{array}\right\}
$$

(where $\Omega$ is a convex polygon in $\mathbf{R}^{2}$ with boundary $\Gamma$, and $\partial w / \partial n$ is the outward normal derivative along $\Gamma$ ). Problem (5.1) can be regarded as a "model" for the free vibrations of an homogeneous isotropic thin plate clamped along its edges. We discuss the use of two finite element methods of mixed type, Miyoshi's and Hermann-Johnson's, and we derive the dependence of our previous results on the particular approximation parameters and true eigenvalues.

Let $r$ denote the largest exponent in $N \cup\{+\infty\}$ such that the eigenfunctions $\left\{w_{l}\right\}_{l=1}^{\infty}$ of Problem (5.1) satisfy

$$
w_{l} \in H_{0}^{2}(\Omega) \cap H^{r}(\Omega), \quad \forall l \in N_{*}
$$

We recall that $r$ depends on the angles formed by the sides of $\Omega$, and in general we have $r<+\infty$ (see Kondrat'ev [13] for the regularity of 4th order steadystate problems, and Merigot [14] for the regularity of 2nd order eigenvalues problems).

Throughout this section, we shall use the classical Sobolev spaces with the following notations

$$
|v|_{m, \Omega}^{2}=\int_{\Omega} \sum_{|\alpha|=m}\left|\partial^{\alpha} v\right|^{2} d x ; \quad\|v\|_{m, \Omega}^{2}=\sum_{i=0}^{m}|v|_{i, \Omega}^{2} .
$$




\section{(A) Miyoshi's Method}

We set

$$
\begin{gathered}
V=\left\{\underset{\sim}{v}=\left\{v_{i j}\right\}_{1 \leqq i, j \leqq 2} \mid v_{i j} \in L^{2}(\Omega) \text { and } v_{12}=v_{21}\right\}, \\
\tilde{V}=\left\{\underset{\sim}{v} \in V \mid v_{i j} \in H^{1}(\Omega)\right\}, \quad W=H_{0}^{2}(\Omega), \quad \tilde{W}=H_{0}^{1}(\Omega), \\
H=L^{2}(\Omega) ; a(\underset{\sim}{u}, \underset{\sim}{v})=\int_{\Omega} u_{i j} v_{i j} d x, \quad b(\underset{\sim}{v}, \varphi)=-\int_{\Omega} v_{i j} \frac{\partial^{2} \varphi}{\partial x_{i} \partial x_{j}} d x, \\
\tilde{b}(\underset{\sim}{v}, \varphi)=\int_{\Omega} \frac{\partial v_{i j}}{\partial x_{j}} \frac{\partial \varphi}{\partial x_{i}} d x,
\end{gathered}
$$

where a repeated subscript implies summation over 1,2 . If we define

$$
\psi=w \quad \text { and } \quad \underset{\sim}{u}=\left\{u_{i j}\right\}=\left\{\frac{\partial^{2} w}{\partial x_{i} \partial x_{j}}\right\}
$$

it is known that Problem (5.1) is equivalent to Problem (EP).

We then establish a uniformly regular family of triangulations $\mathscr{T}_{h}$ of $\bar{\Omega}$ with triangles $K$ whose sides are less that $h$. If $k \geqq 2$ is a fixed integer, set

$$
\left.\begin{array}{c}
\tilde{V}_{h}=\left\{\underline{v}_{h} \in \tilde{V} \mid \forall K \in \mathscr{T}_{h},\left(v_{h}\right)_{i j \mid K} \in P_{k} \text { for } 1 \leqq i, j \leqq 2\right\}, \\
W_{h}=\left\{\varphi_{h} \in \tilde{W} \mid \forall K \in \mathscr{T}_{h}, \varphi_{h \mid K} \in P_{k}\right\}
\end{array}\right\}
$$

and consider the approximation $(\mathrm{EP})_{h}$ of problem (5.1). It is easy to check that all the hypotheses in paragraphs 1 and 2 are verified (see BrezziRaviart [8], § 3).

In particular, let $(\underset{\sim}{\sim}, \psi) \in V \times W$ be the solution of (1.7) for an arbitrary $g \in H$ and let $\left(\tilde{\sim}_{h}, \psi_{h}\right) \in V_{h} \tilde{\times} W_{h}$ be its corresponding approximation, i. e. the solution of (2.4); if we assume that $w \in H^{r^{\prime}}(\Omega)$ with $r^{\prime}$ integer $\geqq 3$ and recall (5.3), we get the estimate

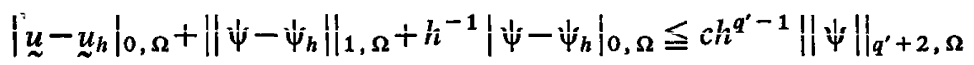

with $c>0$ independent of $h$ and $q^{\prime}=\min \left(k, r^{\prime}-2\right)$.

As a consequence, by standard estimates for interpolation errors (2.12)(2.15), we see that assumption (3.9) is verified, hence by (3.10), (3.7), (3.8) we get

$$
\lambda_{l}^{h}-\lambda_{l} \leqq c_{l} h^{q-1} \max _{\psi \in \bar{\Sigma}_{l}}\|\psi\|_{q+2, \Omega}
$$

with $q=\min (k, r-2)$ and $c_{l}$ depending on $l$. If $r \geqq 4$ then the right side is at least $O(h)$ as $h \rightarrow 0$. On the other hand we have by (5.5):

$$
\left|T \varphi_{h}-T_{h} \varphi_{h}\right|_{0, \Omega} \leqq c h^{q^{\prime}}\left\|T \varphi_{h}\right\|_{q^{\prime}+2, \Omega}
$$

vol. $12, \mathrm{n}^{\circ} 1,1978$ 
and since certainly $T \varphi_{h} \in H_{0}^{2}(\Omega) \cap H^{3}(\Omega)$ with $\left\|T \varphi_{h}\right\|_{3, \Omega} \leqq c\left|\varphi_{h}\right|_{0, \Omega}$, we can take $q^{\prime}=1$ and obtain by $(3.11)$ :

$$
\lambda_{l}-\lambda_{l}^{h} \leqq c \lambda_{l} \lambda_{l}^{h} h
$$

REMARK 5.1: If $\varphi_{h}$ is any trial function in $W_{h}$, we cannot have much more than $T \varphi_{h} \in H^{3}(\Omega)$, so that (5.7) is the best result we can derive from (3.16) even if $k$ is large. Hovever (5.7) together with (5.6) is enough to guarantee that $\left|\lambda_{l}-\lambda_{l}^{h}\right|=O(h)$ as $h \rightarrow 0$, hence assumption (A) of paragraph 4 is verified, and we can get an optimal bound for $\left|\lambda_{l}-\lambda_{l}^{h}\right|$ by means of $\left|\psi_{l}-\psi_{l}^{h}\right|_{H}$. For Problem (5.1) we have $d_{l} \simeq c_{\Omega} \lambda_{l}^{1 / 2}$ as $l \rightarrow+\infty$ (see e. g. Goulaouic [12]), so that by (4.6) and (5.5) we easily obtain:

$$
\left|\psi_{l}-\psi_{l}^{h}\right|_{0, \Omega} \leqq c \lambda_{l}^{1 / 2} h^{q}\left\|\psi_{l}\right\|_{q+2, \Omega} .
$$

This implies that $\psi_{l}^{h} \rightarrow \psi_{l}$ in $H$ as $h \rightarrow 0$; since $P_{h} \psi_{l} \rightarrow \psi_{l}$ in $H$ as $h \rightarrow 0$, we have $\left(P_{h} \psi_{i}, \psi_{i}^{h}\right)_{H} \rightarrow 1$ as $h \rightarrow 0$, hence $\left(P_{h} \psi_{l}, \psi_{i}^{h}\right)_{H} \geqq 1 / 2$ for small $h$. Moreover the hypothesis of uniformly regular mesh implies $S(h) \leqq c h^{-1}$, for a suitable constant $c$. Then, by (3.13), (5.5) and (5.8) we can derive our main estimate for eigenvalues

$$
\left|\lambda_{l}-\lambda_{l}^{h}\right| \leqq c \lambda_{l}^{3 / 2} h^{2(q-1)}\left\|\psi_{l}\right\|_{q+2, \Omega}^{2}
$$

with $c$ independent of $h$ and $l$.

REMARK 5.2: We observe that the exponent of $h$ in (5.9) is optimal, in the sense that - as in usual elliptic eigenvalue problems - it is twice the order of convergence of the corresponding steady-state problem.

REMARK 5.3: Bounds for the norm $\left\|\psi_{l}\right\|_{q+2, \Omega}$ in terms of the eigenvalue $\lambda_{l}$ require regularity results for the solution of the 4 th order Dirichlet boundary value problem in domains with angular points (see Kondrat'ev [13] and Merigot [15]); such bounds do not seem easy to be obtained since the basic estimate $\|\psi\|_{s+4, \Omega} \leqq c_{s}\left\|\Delta^{2} \psi\right\|_{s, \Omega}$ is no longer true for all positive $s$ when the boundary is not smooth. If - according to the value of $q$ and the size of the angles of $\Omega$-we were allowed to apply such an estimate iteratively, and (possibly) the interpolation formula $\|\psi\|_{s, \Omega} \leqq\|\psi\|_{4, \Omega}^{1-9}\|\psi\| \|_{0, \Omega}^{\vartheta}$ for $0 \leqq s \leqq 4$ and $4(1-\vartheta)=s$, then we could obtain the following bound

$$
\left\|\psi_{l}\right\|_{q+2, \Omega} \leqq c_{q} \lambda_{l}^{(q / 4)+(1 / 2)}
$$

Finally, we can obtain the estimate for the other norms of our eigenfunctions. We use estimate (3.12) instead of (5.9) because it involves $\left\|\psi_{l}\right\|_{q+2, \Omega}$ rather than $\left\|\psi_{l}\right\|_{q+2, \Omega}^{2}$. By (4.10), (4.11), (5.5) and (5.8) we obtain

$$
\left|\underset{\sim}{u_{l}}-{\underset{\sim}{u}}_{l}^{h}\right|_{0, \Omega}+\left\|\psi_{l}-\psi_{l}^{h}\right\|_{1, \Omega} \leqq c \lambda_{l}^{3 / 2} h^{q-1}\left\|\psi_{l}\right\|_{q+2, \Omega} .
$$


REMARK 5.4: This result is optimal, according to what we noticed in Remark 5.2.

\section{(B) Hermann-Johnson's Method}

We define the spaces $V, W, H$ and the forms $a(u, v), b(v, \varphi)$ as in (5.2); again the relations among $w, \psi$ and $\underset{\sim}{u}$ are given in $(\tilde{5} .3)$.

We establish a regular (but not necessarily uniform) family of triangulations $\mathscr{T}_{h}$ of $\bar{\Omega}$ with triangles $K$ whose sides are less than $h$. Given a triangle $K \in \mathscr{T}_{h}$ and a tensor-valued functior $v=\left\{v_{i j}\right\}$ with $v_{i j} \in H^{1}(K)$, $1 \leqq i, j \leqq 2$ and $v_{12}=v_{21}$, we define

$$
\left.\begin{array}{l}
M_{n}(\underset{\sim}{v})=v_{i j} n_{i} n_{j} \in \mathrm{L}^{2}(\partial K) \\
M_{n t}(\underset{\sim}{v})=v_{i j} n_{j} t_{i} \in \mathrm{L}^{2}(\partial K)
\end{array}\right\}
$$

where $n=\left(n_{1}, n_{2}\right)$ is the unit outward normal and $t=\left(t_{1}, t_{2}\right)$ is the unit clockwise tangent along the boundary $\partial K$ of $K$ (see Brezzi-Raviart [8], paragraph 4 for more details). We set

$$
\begin{gathered}
\tilde{V}=\left\{\underset{\sim}{v} \in V \mid \forall K \in \mathscr{T}_{h}, v_{i j \mid K} \in H^{1}(K), 1 \leqq i, j \leqq 2\right. \\
\text { and } \left.M_{n}(\underset{\sim}{v}) \text { is continuous at the interelement boundaries }\right\}, \\
\tilde{W}=W_{0}^{1, p}(\Omega) \quad \text { with } p>2, \\
\tilde{b}(\underset{\sim}{v}, \varphi)=\sum_{K \in \mathscr{T}_{h}}\left\{\int_{K} \frac{\partial v_{i j}}{\partial x_{j}} \frac{\partial \varphi}{\partial x_{i}} d x-\int_{\partial K} M_{n t}(\underset{\sim}{v}) \frac{\partial \varphi}{\partial t} d t\right\}
\end{gathered}
$$

where $\int_{\partial K}$ stands for the duality pairing between $H^{1 / p}(\partial H)$ and $H^{-1 / p}(\partial K)$. For any fixed integer $k \geqq 1$, we define

$$
\begin{aligned}
& V_{h}=\left\{\underline{v}_{h} \in \tilde{V} \mid \forall K \in \mathscr{T}_{h},\left(v_{h}\right)_{i j \mid K} \in P_{k_{-1}} \text { for } 1 \leqq i, j \leqq 2\right\}, \\
& W_{h}=\left\{\varphi_{h} \in W_{0}^{1, p}(\Omega) \mid \forall K \in \mathscr{T}_{h}, \varphi_{h \mid K} \in P_{k}\right\} .
\end{aligned}
$$

Again all hypotheses in paragraphs 1 and 2 -in particular condition (3.4)are verified (see Brezzi-Raviart [8], §4). We remark (id., § 2, Remark 1) that hypothesis $(1.26)$ must be replaced by the following

$$
\sup _{\underset{\sim}{v} \in \tilde{V}} \frac{\tilde{b}(\underset{\sim}{v}, \varphi)}{\|\underset{\sim}{v}\| \tilde{V}} \geqq \tilde{\beta}\|\varphi\|_{1, \Omega}, \quad \forall \varphi \in \tilde{W}
$$

Hence, if $(u, \psi) \in V \times W$ is the solution of (1.7) for an arbitrary $g \in H$ and $\left(u_{h}, \psi_{h}\right) \in \tilde{V_{h}} \times W_{h}$ is the corresponding approximation, assuming $w \in H^{r^{\prime}}(\Omega)$

vol. $12, \mathrm{n}^{\circ} 1,1978$ 
with $r^{\prime}$ integer $\geqq 3$ and recalling (5.3), we have the estimate (BrezziRaviart [8], Th. 8)

$$
\left|\underset{\sim}{u}-{\underset{\sim}{h}}_{h}\right|_{0, \Omega}+\left\|\psi-\psi_{h}\right\|_{1, \Omega}+h^{-1}\left|\psi-\psi_{h}\right|_{0, \Omega} \leqq c h^{q^{\prime}}\|\psi\|_{q^{\prime}+2, \Omega}
$$

with $c>0$ independent of $h$ and $q^{\prime}=\min \left(k, r^{\prime}-2\right)$,

Let us now look at the interpolation errors (2.12)-(2.15). From Lemma 4 of Brezzi-Raviart [8], we get

$$
\varepsilon^{h}(\underset{\sim}{v}) \leqq c h^{k}|\underset{\sim}{v}|_{k, \Omega}, \quad \forall \underset{\sim}{v} \in \tilde{V} \text { regular enough }
$$

while, with the same proof of Lemma 4 we obtain

$$
\tilde{\varepsilon}^{h}(\underset{\sim}{v})=\left\|\underset{\sim}{v}-\pi_{h} \underset{\sim}{v}\right\|_{1, \Omega} \leqq c h^{k-1}|\underset{\sim}{v}|_{k, \Omega}, \quad \forall \underset{\sim}{v} \in \tilde{V} \text { regular enough }
$$

with $c>0$ independent of $h$, Moreover the definition (2.15) of $\tilde{\varepsilon}^{h}(\varphi)$ must be replaced by the following (id., $\S 2$, Remark 1):

$$
\tilde{\varepsilon}^{h}(\varphi)=\left\|\varphi-r_{h} \varphi\right\|_{1, \Omega}
$$

Now (3.10), (3.7), (3.8), (5.14) lead us to

$$
\lambda_{l}^{h}-\lambda_{l} \leqq c_{l} h^{q} \max _{\psi \in \bar{\Sigma}_{l}}\|\psi\|_{q+2, \Omega}
$$

with $q=\min (k, r-2)$ and $c_{l}$ depending on $\lambda_{l}$. Since $r \geqq 3$, the right side is certainly $O(h)$ as $h \rightarrow 0$, On the other hand, by (3.11) and (5.14) with $q^{\prime}=1$, we get

$$
\lambda_{l}-\lambda_{l}^{h} \leqq c \lambda_{l} \lambda_{l}^{h} h
$$

so that $\left|\lambda_{l}-\lambda_{l}^{h}\right|=O(h)$ at least. Hence (4.6) and (5.14) give us

$$
\left|\psi_{l}-\psi_{l}^{h}\right|_{0, \Omega} \leqq c \lambda_{l}^{1 / 2} h^{q+1}\left\|\psi_{l}\right\|_{q+2, \Omega}
$$

while estimate $(3.13$ bis $)$ with (5.14), (5.15), (5.16) yield

$$
\left|\lambda_{l}-\lambda_{l}^{h}\right| \leqq c \lambda_{l}^{3 / 2} h^{2 q-1}\left\|\psi_{l}\right\|_{q+2, \Omega}
$$

with $c$ independent of $h$ and $l$. Again $\left\|\psi_{l}\right\|_{q+2, \Omega, \Omega}$ could be bounded as in $(5.10)$.

REMARK 5.5: The order of convergence in (5.20) is not optimal, since the basic estimate $(3.13 \mathrm{bis})$ involves the error $\tilde{\varepsilon}^{h}(v)$, which is given by $(5.16)$. Thus we can get optimality if we replace $P_{k-1}$ with $P_{k}$ in the definition of $V_{h}$ (leaving unchanged the definition of $W_{h}$ ).

Finally, by (4.10), (4.11), (3.12), (5.14) and (5.19) we obtain:

$$
\left|{\underset{\sim}{u}}_{l}-{\underset{\sim}{l}}_{l}^{h}\right|_{0, \Omega}+\left\|\psi_{l}-\psi_{l}^{h}\right\|_{1, \Omega} \leqq c \lambda_{l}^{3 / 2} h^{q}\left\|\psi_{l}\right\|_{q+2, \Omega} .
$$




\section{REFERENCES}

1. I.BABUšKa, The Finite Element Method with Lagrangian Multipliers, Num. Math., 20, 1973, pp. 179-192.

2. I. BABUŠKA and A. K. AzIZ, Survey Lectures on the Mathematical Foundations of the Finite Element Method in The Mathematical Foundations of the Finite Element Method with Applications to Partial Differential Equations, A. K. Azız, Ed., Academic Press, New York 1972, pp. 3-359.

3. I. Babuška, J. T. Oden and J. K. LeE, Mixed-Hybrid Finite Element Approximations of Second Order Elliptic Boundary-Value Problems, TICOM Report 75-7, University of Texas at Austin, 1975.

4. I. BABUŠKA and J. E. OsBoRn, Numerical Treatment of Eigenvalue Problems for Differential Equations with Discontinuous Coefficients, Technical Note BN-853, University of Maryland, College Park, 1977.

5. G. BIRKhoff, C. DE Boor, B. SwarTz and B. Wendroff, Rayleigh-Ritz Approximation by Piecewise Cubic Polynomials, S.I.A.M. J. Num. Anal., 13, 1973, pp. 188-203.

6. J. H. BRAMBLE and J. E. OsBoRN, Rate of Convergence Estimates for Nonselfadjoint Eigenvalue Approximation, Math. of Comp., vol. 27, No. 123, 1973, pp. 525-549.

7. F. BrezzI, On the Existence, Uniqueness and Approximations of Saddle-Point Problems Arising from Lagrangian Multipliers, R.A.I.R.O., R 2, aout 1974, pp. 129-151.

8. F. Brezzi and P. A. Raviart, Mixed Finite Element Methods for 4th Order Elliptic Equations, Rapport Interne No. 9, C.M.A. École Polytechnique, Palaiseau, 1976.

9. P. G. Ciarlet and P. A. Raviart, A Mixed Finite Element Method for the Biharmonic Equation Symposium on Mathematical Aspects of Finite Elements in Partial Differential Equations, C. DE BooR, Ed., Academic Press, New York, 1974, pp. 125-145.

10. G. FIx, Eigenvalue Approximation by the Finite Element Method, Adv. in Math., 10, 1973, pp. 300-316.

11. R. GLowinski, Approximations externes, par éléments finis de Lagrange d'ordre iin êt deuix, dii problème de Dirichlet poutr l'opérateur biharmonique. Méthode itérative de résolutions des problèmes approchés, Topics in Numerical Analysis, J. J. H. Miller, Ed., Academic Press, London, 1973, pp. 123-171.

12. C. GoulaоUс, Valeurs propres de problèmes aux limites irréguliers : applications, in Spectral Analysis, C.I.M.E. Session 1973, Cremonese, Rome, 1974, pp. 80-140.

13. V. A. Kondrat'ev, Boundary Problems for Elliptic Equations in Domains with Conical or Angular Points, Trans. Moscow Math. Soc., vol. 16, 1967, pp. 227-313.

14. M. Merigot, Régularité des fonctions propres du laplacien dans un cône, C. R. Acad. Sc., Paris, 279, série A, 1974, pp. 503-505.

15. M. Merigot, Solutions en norme $L^{p}$ des problèmes elliptiques dans des polygônes plans, Thèse à l'Université de Nice, 1974.

16. J. E. OsBoRn, Spectral Approximation for Compact Operators, Math. of Comp., 29, 1975, pp. 712-725.

vol. $12, \mathrm{n}^{\circ} 1,1978$ 
17. P. A. Raviart and J. M. Thomas, A Mixed Finite Element Method for and Order Elliptic Problems, in Mathematical Aspects of Finite Element Methods, Lecture Notes in Mathematics, No. 606, Springer-Verlag, Berlin-HeidelbergNew York, 1977, pp. 292-315.

18. J. M. Thомas, Sur l'analyse numérique des méthodes d'éléments finis hybrides et mixtes, Thèse à l'Université Pierre-et-Marie-Curie, Paris, 1977.

19. G. Strang and G. FIX, An Analysis of the Finite Element Method, Prentice Hall, New York, 1973. 